\title{
Long-Term Effects of Safinamide on Dyskinesia in Mid- to Late-Stage Parkinson's Disease: A Post-Hoc Analysis
}

\author{
Carlo Cattaneo ${ }^{\mathrm{a}, *}$, R. La Ferla ${ }^{\mathrm{a}}$, Erminio Bonizzoni $^{\mathrm{b}}$ and Marco Sardina ${ }^{\mathrm{c}}$ \\ ${ }^{a}$ Department of Medical, Zambon SpA, Bresso, Italy \\ b Department of Clinical Science and Community, Section of Medical Statistics and Biometry “GA Maccacaro”, \\ University of Milan, Italy \\ ${ }^{\mathrm{c}}$ Department of $R \& D$, Zambon $S p A$, Bresso, Italy
}

\begin{abstract}
.
Background: Safinamide is a novel $\alpha$-aminoamide with dopaminergic and non-dopaminergic properties developed as adjunctive therapy for patients with PD. Results from a 24-month double-blind controlled study suggested that as add-on to levodopa (and other PD medications) the benefits of safinamide on dyskinesia may be related to severity of dyskinesia at baseline.

Objective: This post-hoc analysis further characterized the effects of safinamide on dyskinesia in mid- to late-stage PD patients. Methods: Patients were stratified by the presence or absence of dyskinesia at baseline, and by whether or not the dose of levodopa had been changed during the 24-month treatment period. Differences between safinamide and placebo were evaluated using the Wilcoxon rank-sum test.

Results: For the overall treated population (with or without baseline dyskinesia), safinamide $100 \mathrm{mg} /$ day significantly improved the dyskinesia rating scale score, compared with placebo, in the subgroup of patients with no change in levodopa dose $(p=0.0488)$. For patients with baseline dyskinesia, improvements over placebo were also significant $(p=0.0153)$ in patients with or without changes in levodopa dose, and nearly significant $(p=0.0546)$ in patients with no change in levodopa dose, suggesting that these improvements were not due to levodopa dose reductions.

Conclusions: While no statistically significant difference in mean DRS scores was seen between safinamide and placebo in the original study population, the present post-hoc analysis helps to provide a meaningful interpretation of the long-term effects of safinamide on dyskinesia. These results may be related to safinamide state- and use-dependent inhibition of sodium channels and stimulated glutamate release, and are unlikely due to reduced dopaminergic stimulation.
\end{abstract}

Keywords: Parkinson's disease, levodopa, safinamide, dyskinesia

\section{INTRODUCTION}

Levodopa (L-dopa) remains the mainstay for treatment of the motor symptoms of Parkinson's disease (PD) [1]. However, as the disease progresses, initial

*Correspondence to: Carlo Cattaneo DBSc CAAAN, Corporate Medical Advisor CNS, Zambon SpA, via Lillo del Duca, 10, 20091 Bresso (Milan), Italy. Tel.: +39 02 66524299; Mobile: +39 347 6174245; Fax: +39 02 66524887; E-mail: carlo.cattaneo@ zambongroup.com. therapy becomes less effective and motor complications, including dyskinesia and fluctuations, eventually develop [2]. About $40 \%$ of patients will experience motor fluctuations and dyskinesia after 4-6 years of levodopa therapy [3]. Unfortunately, levodopa-induced dyskinesia remains difficult to treat: the efficacy of levodopa decreases with time and disease symptoms recur with subsequent impairment on motor function and quality of life [4]. There are different strategies usually adopted to control motor complications: use 
of slow-release levodopa formulations, or start with an adjunctive therapy such as a dopamine agonist, a COMT-inhibitor or a MAO-B inhibitor [5, 6]. However, treatment of PD should notbe limited to simply targeting the dopaminergic system [7]. In particular, overactive glutamate transmission plays a role in the progression of PD [8-10]. Targeting non-dopaminergic systems is thus a complementary approach to improve and control such motor complications, while maintaining the efficacy of levodopa [7]. A variety of medications may be considered in the attempt to reduce dyskinesia, but only amantadine has a weak evidence-based recommendation for dyskinesia, with an effect that is generally considered to be modest and relatively short-lasting [4].

Safinamide (Xadago ${ }^{\mathrm{TM}}$, Zambon S.p.A.) is a novel $\alpha$-aminoamide that has been developed as adjunctive therapy for PD as add-on to dopamine therapy in early PD [11-13] and as add-on to levodopa in mid- to latestage PD [14, 15]; safinamide 50 and $100 \mathrm{mg}$ /day has been approved in EU for the treatment of adult patients with idiopathic PD as add-on therapy to a stable dose of levodopa, alone or in combination with other PD medicinal products, in mid-to late-stage fluctuating patients.

Safinamide is a unique compound with a novel dual mechanism of action (dopaminergic and nondopaminergic) that includes reversible and highly selective monoamine oxidase-B (MAO-B) inhibition, sodium channel blockade and N-type calcium channel modulation. The sodium channel inhibition is both concentration- and state-dependent (different potency at different states of the channel), thus inhibiting the excessive presynaptic release of excitotoxic amino acids like glutamate without influencing the physiological activity [16-20]. Safinamide does not affect L-type calcium channels (no effects on blood pressure or heart rate) $[16,17,21,22]$, is well tolerated with a favorable side effect profile, has no major drug-drug interactions, and no diet restrictions [20, 23, 24].

As add-on to levodopa, safinamide improved motor symptoms and controlled motor complications in the short term, maintaining the benefits in the long term. Results from a long-term (24 months) double-blind controlled study (study 018) suggest that safinamide significantly increased daily ON time without increasing the risk of developing troublesome dyskinesia, and significantly improved the activities of daily living and the quality of life [15]. For the overall population of study 018 , the rather low baseline dyskinesia score values allowed little room for improvement, and no statistically significant difference in DRS scores was seen between safinamide and placebo; in fact, a significant improvement in favor of safinamide was seen only in patients with moderate to severe dyskinesia at baseline. A post-hoc analysis of study 018 is presented herein in order to summarize the full set of results in order to provide a clinically-meaningful interpretation about the long-term effects of safinamide on the evolution of dyskinesia in mid- to late-stage PD patients.

\section{MATERIALS AND METHODS}

Trial 018 was a multicenter, multinational, doubleblind, placebo-controlled, extension study of a pivotal 24-week Phase III trial 016 [14] in 669 mid- to late-stage PD fluctuating patients on a stable levodopa dose. Patients were included in the extension study (study 018) if they had completed the initial study, were treatment compliant and willing to continue, or if they had discontinued but had completed the scheduled efficacy evaluations at weeks 12 and 24. They were excluded if they had experienced clinically significant adverse events or shown clinically significant deterioration in motor symptoms during study 016. Both protocols and patient materials were approved by Independent Ethics Committees and Health Authorities in all three participating countries (India, Romania, Italy). All patients signed an informed consent form and the study was conducted according to the Declaration of Helsinki. Study 018 is registered on Clinicaltrials.gov (NCT01187966). After completing the initial treatment period, patients willing to participate in the extension phase continued on their randomized study medications and were followedup during an additional period of 18 months [15]. Safinamide (50 or $100 \mathrm{mg} /$ day p.o.) or placebo were given as add-on to levodopa and other PD therapies (dopamine agonists, COMT inhibitors, amantadine, and/or anticholinergics). In case of motor symptom deterioration, dose increases of levodopa or additional PD drugs, except MAO-B inhibitors, were permitted. The levodopa dose could also be decreased based on patient conditions or the occurrence of adverse events.

The primary variable of study 018 was the mean change from baseline (study 016 start) to study end in the total dyskinesia rating scale (DRS) score during ON time. The DRS is an easy-to-use and practical tool used to rate the severity of dyskinesia and to identify the most disabling dyskinesia [25]. The primary analysis estimated the mean difference between placebo and safinamide in the primary variable. 


\section{Study design and statistical analysis}

The present study was a post-hoc analysis of study 018 that evaluated the categorical changes in DRS scores at the end of study 018 after stratifying patients based on the presence or absence of dyskinesia (DRS score $>0$ or DRS score $=0$, respectively) at baseline, and by additional subgroups based on whether or not the dose of levodopa had been changed during the entire treatment period of 24 months. Proportions of patients with categorical changes in DRS scores were evaluated, since DRS score distributions were ill-conditioned (because of skewness and the presence of outliers). Changes in DRS scores were classified as "decreased", "unchanged", and "increased", and patients were classified according to changes in DRS scores during the study using a 3-point ordinal scale ( $1=$ improved, DRS score decreased; $2=$ stable; $3=$ worsened, DRS score increased). The comparisons of active treatments (safinamide $100 \mathrm{mg}$ and safinamide $50 \mathrm{mg}$ ) versus placebo were performed using the Wilcoxon rank-sum test for independent samples. Since in the original protocol of study 018 [23] the multiplicity issue (over treatment groups) was handled by using a pre-specified "sequence of comparisons" approach [26], no adjustment of type I error was needed for the multiple comparisons. All statistical analyses were performed using SAS software version 9.4.

\section{RESULTS}

In the original 018 trial, safinamide maintained a statistically significant improvement in ON time without dyskinesia over 2 years of treatment, confirming the results obtained in the initial 6-month study 016 . After 2 years, there was a decrease from baseline in mean DRS score during ON time of $31 \%$ and $27 \%$ with safinamide 50 and $100 \mathrm{mg} /$ day, respectively, compared to a decrease of only $3 \%$ with placebo. However, the primary endpoint was not met [least-squares mean difference vs. placebo, -0.51 score points $(95 \% \mathrm{CI}:-1.32$ to $0.29, p=0.2125$ ) for safinamide $50 \mathrm{mg}$, and -0.59 (95\% CI: -1.40 to $0.21, p=0.1469$ ) for safinamide $100 \mathrm{mg}$ [ [15].

The proportions of patients with reduced levodopa dose were higher in both safinamide groups compared to placebo, while the proportions of patients with an increase in levodopa dose had an opposite trend (Fig. 1). Over the study period, the overall mean dose of levodopa increased in the placebo arm and in the safinamide $50 \mathrm{mg} /$ day group, and decreased in

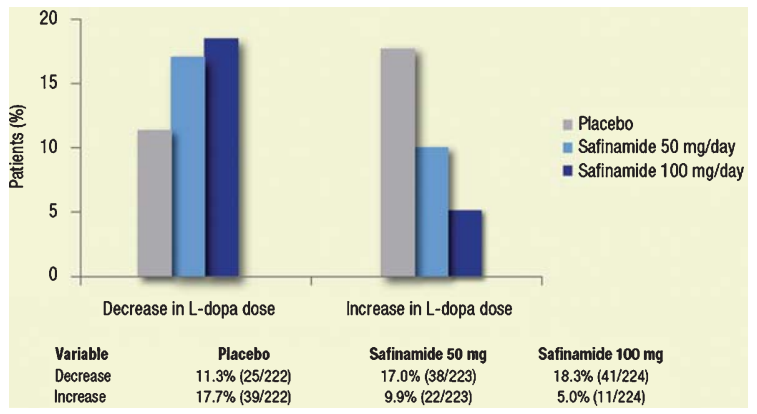

Fig. 1. Proportions of patients with changes in L-dopa dose over 2 years [15].

Table 1

Changes in the mean $( \pm \mathrm{SD})$ dose of L-dopa from baseline to 24 months [15]

\begin{tabular}{ccc}
\hline Placebo & Safinamide & Safinamide \\
$(N=222)$ & $50 \mathrm{mg} /$ day & $100 \mathrm{mg} /$ day \\
& $(N=223)$ & $(N=224)$ \\
\hline
\end{tabular}

\begin{tabular}{llll}
\hline Baseline (mg/day) & $618.5 \pm 335.7$ & $621.4 \pm 329.7$ & $579.6 \pm 310.0$
\end{tabular} \begin{tabular}{llll} 
Month 24 (mg/day) & $650.6 \pm 338.0$ & $635.2 \pm 410.4$ & $556.0 \pm 381.9$ \\
\hline
\end{tabular}

the safinamide $100 \mathrm{mg} /$ day group (Table 1). Such dose changes do not appear to be clinically relevant [15].

An already published initial post-hoc analysis of the changes in DRS in patients with moderate to severe dyskinesia at baseline (DRS total score $>4$, $n=242 / 594$ ) showed a more consistent decrease in DRS scores from baseline to study end in all treatment groups. Notably, the least-squares (LS) mean change in DRS scores from baseline to study end for the safinamide 100-mg group was significantly different from the placebo group [LS mean difference vs. placebo, -1.22 (95\% CI, -2.33 to $-0.11, p=0.0317)$ ] [15].

\section{Post-hoc analysis}

Both doses of safinamide (50 and $100 \mathrm{mg} /$ day) were generally well tolerated over 2 years by patients who were receiving polypharmacy. In the subgroup of patients (with or without dyskinesia at baseline, DRS $\geq 0$ ) with no changes in their levodopa dose during the entire treatment period $(n=431)$, the proportion of those with an improvement (decrease) in DRS scores was higher in both safinamide groups compared with placebo (Fig. 2). Concurrently, the proportion of patients with a worsening (DRS increase) of dyskinesia was lower in both safinamide groups compared with placebo. The difference between safinamide $100 \mathrm{mg}$ and placebo, considering the 3-point ordinal scale (decreased, unchanged, increased), was 


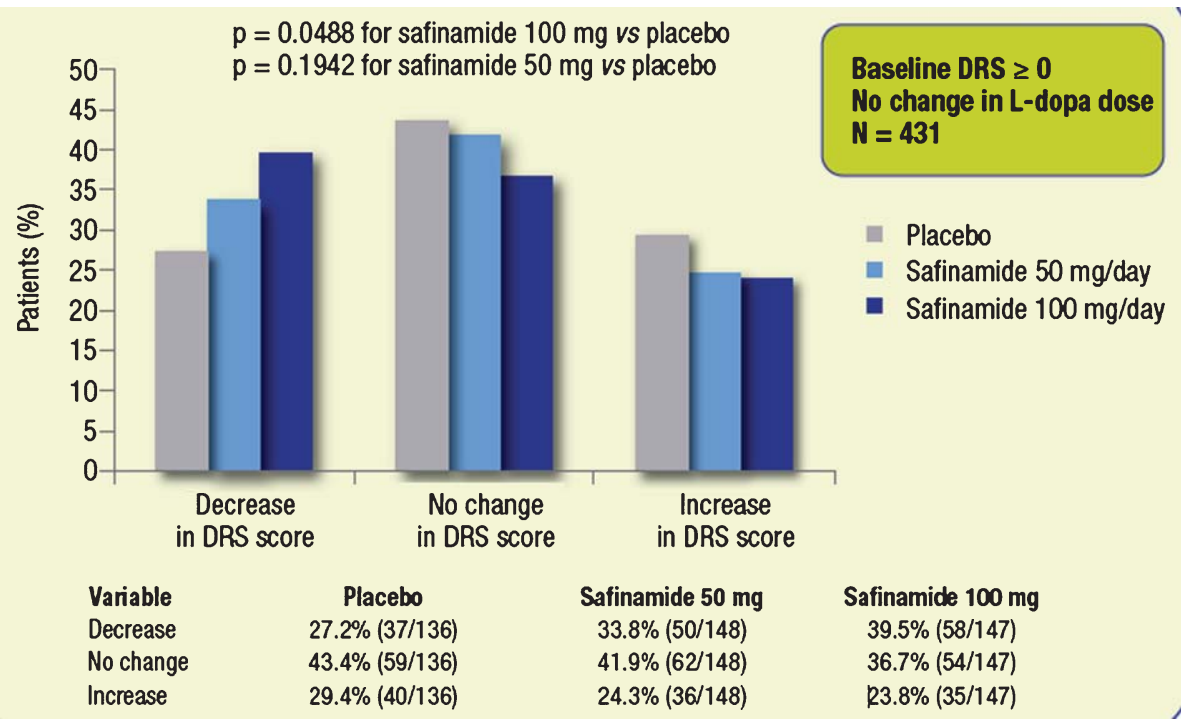

Fig. 2. Proportions of patients with different categorical changes in DRS score (decrease, no change, increase). Subgroups of patients whose dose of L-dopa was not changed throughout the study.

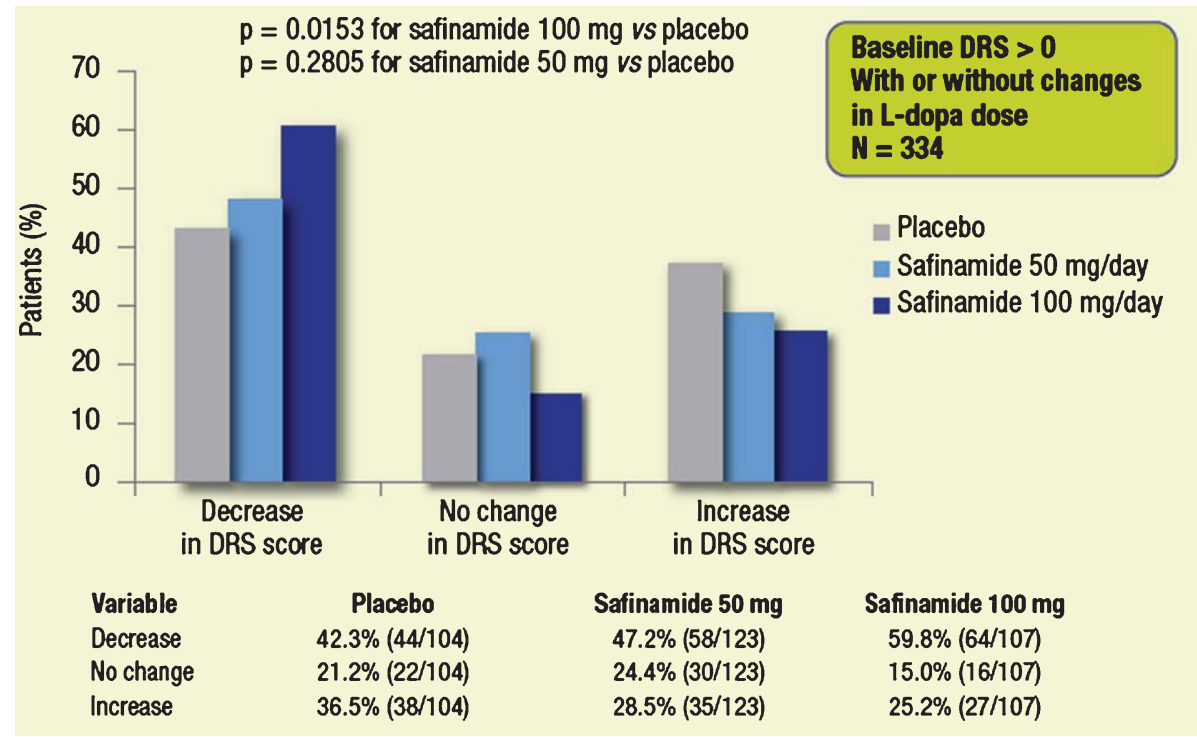

Fig. 3. Proportions of patients with different categorical changes in DRS score (decrease, no change, increase). Subgroups of patients with dyskinesia at baseline.

statistically significant $(p=0.0488)$. This suggests that the beneficial effect of safinamide $100 \mathrm{mg}$ on DRS scores was not dependent on changes in the levodopa dose, as this analysis excluded patients with a dose reduction.

When the subgroup of patients with dyskinesia at baseline (DRS $>0, n=334$ ) was considered (Fig. 3), more patients treated with $100 \mathrm{mg}$ or $50 \mathrm{mg}$ safinamide vs. placebo had a decrease in DRS scores. This finding was paralleled by lower proportions of patients in both safinamide groups who showed an increase in the DRS score compared with placebo. The difference considering the 3-point ordinal scale (decreased, unchanged, increased) between safinamide $100 \mathrm{mg}$ and placebo was statistically significant $(p=0.0153)$. 


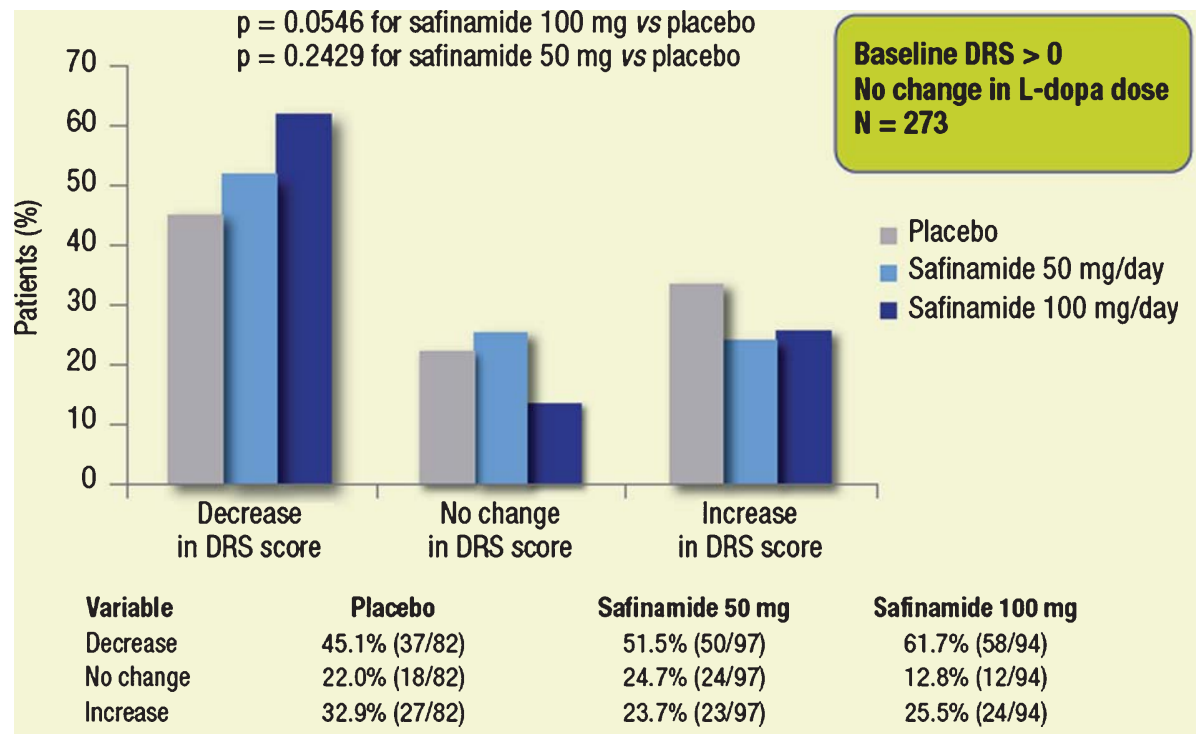

Fig. 4. Proportions of patients with different categorical changes in DRS score (decrease, no change, increase). Subgroups of patients with dyskinesia at baseline, whose dose of L-dopa was not changed throughout the study.

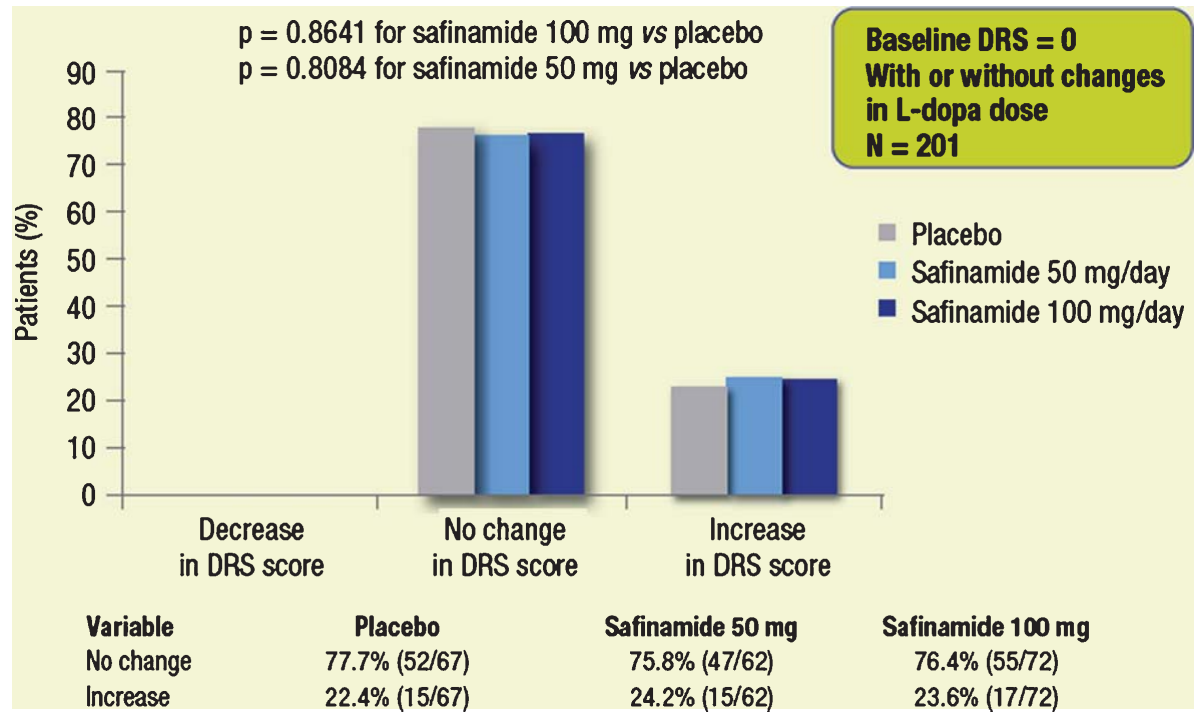

Fig. 5. Proportions of patients with different categorical changes in DRS score (decrease, no change, increase). Subgroups of patients without dyskinesia at baseline.

In the subgroup of patients with dyskinesia at baseline (DRS $>0)$ and no change in L-dopa dose $(n=273)$, the proportion of patients with a decrease in the DRS score was again greater in both safinamide groups compared with placebo, while the proportion of patients with an increase in the DRS score was lower in both safinamide groups vs. placebo (Fig. 4). The distribution difference considering the 3-point ordinal scale (decreased, unchanged, increased) between the safinamide $100 \mathrm{mg}$ and placebo groups showed a trend towards significance $(p=0.0546)$.

Considering the subgroup of patients without dyskinesia at baseline (DRS $=0, n=201$ ), DRS scores remained unchanged throughout the 2-year treatment period for the majority $(\geq 75 \%)$ of patients in all treatment groups; the proportions of patients with 


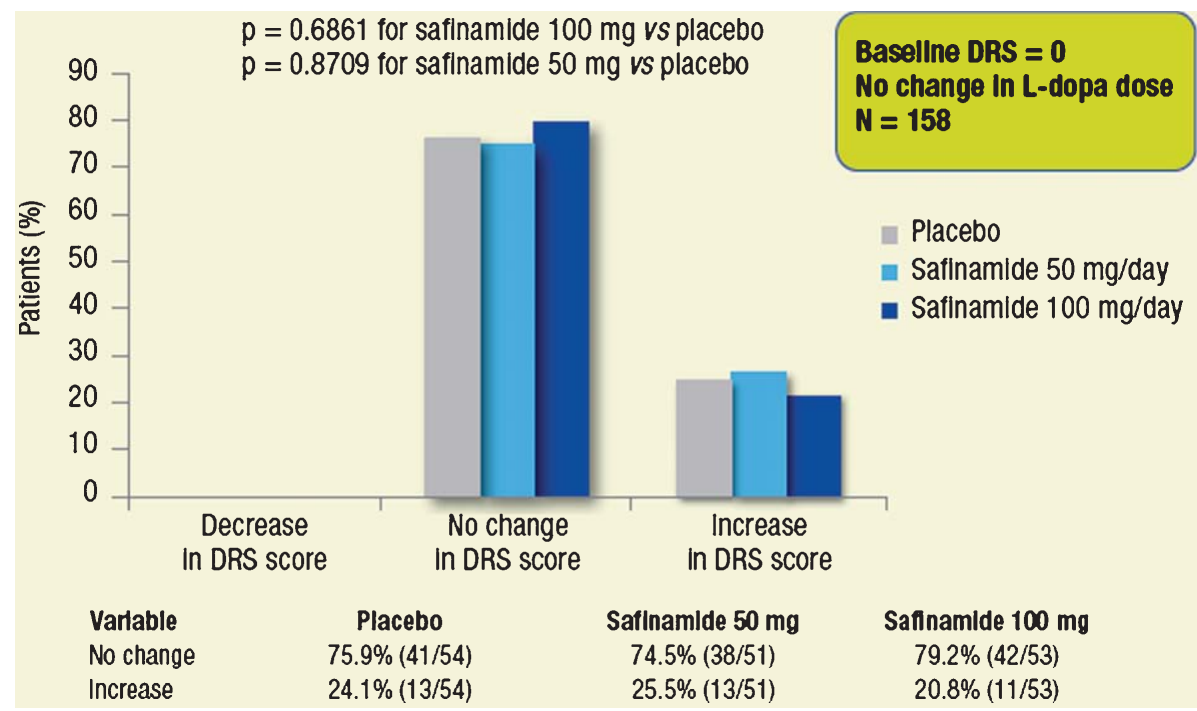

Fig. 6. Proportions of patients with different categorical changes in DRS score (decrease, no change, increase). Subgroups of patients without dyskinesia at baseline, whose dose of L-dopa was not changed throughout the study.

worsening of dyskinesia were low and similar across treatment groups (Fig. 5). A similar trend was seen in the subgroup of patients with no change in L-dopa dose ( $n=158$; Fig. 6). None of the observed differences was statistically significant.

\section{DISCUSSION}

In study 018 , safinamide $100 \mathrm{mg}$ /day was administered as add-on to levodopa and other PD medications in patients with mid- to late-stage PD and motor fluctuations over 2 years of treatment: DRS score mean changes from baseline were not significantly different between safinamide and placebo in the original study 018 analysis, even if scores decreased with safinamide and were almost unchanged with placebo.

In terms of categorical changes of DRS scores (proportions of patients showing worsening, no changes and improvements), the present post-hoc analysis showed that safinamide $100 \mathrm{mg}$ was associated with significant differences compared with placebo in the subgroup of patients with no changes in L-dopa dose, suggesting that these improvements were not due to a reduction in the dose of levodopa, but rather to the effects of safinamide itself. Furthermore, this effect is unlikely to be related to reduced dopaminergic stimulation, as demonstrated by statistically significant effects of safinamide on motor fluctuations (ON and OFF times) over 2 years $[14,15]$.

Several neurotransmitters, in addition to dopamine, contribute to the appearance of levodopa-induced dyskinesia [27], and overactive glutamate transmission has been shown to play a role in the progression of PD [8, 10]. Moreover, altered neurotransmission is regulated by signal transduction cascades involving glutamatergic receptors belonging to the NMDA and AMPA classes $[10,28]$. The results of the present post-hoc analysis may be explained by the blockade of sodium channels and modulation of calcium channels by safinamide, which inhibits altered glutamatergic neurotransmission. At the dose of $100 \mathrm{mg} /$ day, the effective safinamide concentration is expected to be fully active on the glutamate transmission; this may further support the hypothesis that the higher dose is needed to maximize both the dopaminergic and nondopaminergic effects of safinamide.

Safinamide is a unique treatment for $\mathrm{PD}$ that exhibits a combined non-dopaminergic and dopaminergic mode of action. Unlike other drugs that can improve motor functions, safinamide does not worsen dyskinesia: this effect may be related to its dual mechanism, which modulates dopaminergic and glutamatergic pathways. The present post-hoc analysis, in terms of categorical changes of DRS scores (proportions of patients), helps to provide a meaningful interpretation of the long-term effects of safinamide $100 \mathrm{mg} /$ day on dyskinesia.

\section{ACKNOWLEDGMENTS INCLUDING SOURCES OF SUPPORT}

The authors have no acknowledgments to disclose. 


\section{CONFLICT OF INTEREST}

Carlo Cattaneo, Roberto La Ferla, and Marco Sardina are employees at Zambon SpA. Erminio Bonizzoni is a consultant statistician for Zambon SpA.

\section{REFERENCES}

[1] LeWitt PA (2009) Levodopa therapeutics for Parkinson's disease: New developments. Parkinsonism Relat Disord, 15(Suppl 1), S31-S34.

[2] Hauser RA (2009) Levodopa: Past, present, and future. Eur Neurol, 62, 1-8.

[3] Ahlskog JE, \& Muenter MD (2001) Frequency of levodoparelated dyskinesias and motor fluctuations as estimated from the cumulative literature. Mov Disord, 16, 448-458.

[4] Pahwa R, Factor SA, Lyons KE, Ondo WG, Gronseth G, Bronte-Stewart H, Hallett M, Miyasaki J, Stevens J, Weiner WJ, \& Quality Standards Subcommittee of the American Academy of N (2006) Practice parameter: Treatment of Parkinson disease with motor fluctuations and dyskinesia (an evidence-based review): Report of the quality standards subcommittee of the american academy of neurology. Neurology, 66, 983-995.

[5] Fabbrini G, Brotchie JM, Grandas F, Nomoto M, \& Goetz CG (2007) Levodopa-induced dyskinesias. Mov Disord, 22 1379-1389; quiz, 1523.

[6] Hinson VK (2010) Parkinson's disease and motor fluctuations. Curr Treat Options Neurol, 12, 186-199.

[7] Fox SH (2013) Non-dopaminergic treatments for motor control in Parkinson's disease. Drugs, 73, 1405-1415.

[8] Blandini F, Porter RH, \& Greenamyre JT (1996) Glutamate and Parkinson's disease. Mol Neurobiol, 12, 73-94.

[9] Borgohain R, \& Kandadai RM (2013) Safinamide: A novel anti-Parkinsonian drug with multiple actions. Neurodegener Dis Manag, 3, 231-240.

[10] Chase TN, Bibbiani F, \& Oh JD (2003) Striatal glutamatergic mechanisms and extrapyramidal movement disorders. Neurotox Res, 5, 139-146.

[11] Schapira AH, Stocchi F, Borgohain R, Onofrj M, Bhatt M, Lorenzana P, Lucini V, Giuliani R, Anand R, \& Study I (2013) Long-term efficacy and safety of safinamide as addon therapy in early Parkinson's disease. Eur J Neurol, 20, 271-280.

[12] Stocchi F, Arnold G, Onofrj M, Kwiecinski H, Szczudlik A, Thomas A, Bonuccelli U, Van Dijk A, Cattaneo C, Sala P, Fariello RG, \& Safinamide Parkinson's Study G (2004) Improvement of motor function in early Parkinson disease by safinamide. Neurology, 63, 746-748.

[13] Stocchi F, Borgohain R, Onofrj M, Schapira AH, Bhatt M, Lucini V, Giuliani R, Anand R, \& Study I (2012) A randomized, double-blind, placebo-controlled trial of safinamide as add-on therapy in early Parkinson's disease patients. Mov Disord, 27, 106-112.
[14] Borgohain R, Szasz J, Stanzione P, Meshram C, Bhatt M, Chirilineau D, Stocchi F, Lucini V, Giuliani R, Forrest E, Rice P, Anand R, \& Study I (2014) Randomized trial of safinamide add-on to levodopa in Parkinson's disease with motor fluctuations. Mov Disord, 29, 229-237.

[15] Borgohain R, Szasz J, Stanzione P, Meshram C, Bhatt MH, Chirilineau D, Stocchi F, Lucini V, Giuliani R, Forrest E, Rice P, Anand R, \& Study I (2014) Two-year, randomized, controlled study of safinamide as add-on to levodopa in mid to late Parkinson's disease. Mov Disord, 29, 1273-1280.

[16] Caccia C, Maj R, Calabresi M, Maestroni S, Faravelli L, Curatolo L, Salvati P, \& Fariello RG (2006) Safinamide: From molecular targets to a new anti-Parkinson drug. Neurology, 67, S18-S23.

[17] Chazot PL (2007) Safinamide for the treatment of Parkinson's disease, epilepsy and restless legs syndrome. Curr Opin Investig Drugs, 8, 570-579.

[18] Gregoire L, Jourdain VA, Townsend M, Roach A, \& Di Paolo T (2013) Safinamide reduces dyskinesias and prolongs L-DOPA antiparkinsonian effect in parkinsonian monkeys. Parkinsonism Relat Disord, 19, 508-514.

[19] Kulisevsky J (2014) Emerging role of safinamide in Parkinson's disease therapy. Eur Neurolog Review, 9, 3-7.

[20] Muller T (2013) Current status of safinamide for the drug portfolio of Parkinson's disease therapy. Expert Rev Neurother, 13, 969-977.

[21] Caccia C, Salvati P, Rossetti S, \& Anand R (2008) Safinamide: Modulation of dopaminergic and glutamatergic system. Mov Disord, 2008, S22-S23.

[22] Pevarello P, Bonsignori A, Dostert P, Heidempergher F, Pinciroli V, Colombo M, McArthur RA, Salvati P, Post C, Fariello RG, \& Varasi M (1998) Synthesis and anticonvulsant activity of a new class of 2-[(arylalky)amino]alkanamide derivatives. J Med Chem, 41, 579-590.

[23] Cattaneo C, Caccia C, Marzo A, Maj R, \& Fariello RG (2003) Pressor response to intravenous tyramine in healthy subjects after safinamide, a novel neuroprotectant with selective, reversible monoamine oxidase B inhibition. Clin Neuropharmacol, 26, 213-217.

[24] Onofrj M, Bonanni L, \& Thomas A (2008) An expert opinion on safinamide in Parkinson's disease. Expert Opin Investig Drugs, 17, 1115-1125.

[25] Goetz CG, Stebbins GT, Shale HM, Lang AE, Chernik DA, Chmura TA, Ahlskog JE, \& Dorflinger EE (1994) Utility of an objective dyskinesia rating scale for Parkinson's disease: Inter- and intrarater reliability assessment. Mov Disord, 9, 390-394.

[26] Marcus R, Peritz E, \& Gabriel KR (1976) On closed testing procedures with special reference to ordered analysis of variance. Biometrika, 63, 665-660.

[27] Brotchie JM (2005) Nondopaminergic mechanisms in levodopa-induced dyskinesia. Mov Disord, 20, 919-931.

[28] Konitsiotis S, Blanchet PJ, Verhagen L, Lamers E, \& Chase TN (2000) AMPA receptor blockade improves levodopainduced dyskinesia in MPTP monkeys. Neurology, 54, 1589-1595. 